\title{
Plasma fatty acids and the risk of metabolic syndrome in ethnic Chinese adults in Taiwan
}

\author{
Kuo-Liong Chien ${ }^{1,2}$, Chia-Lun Chao ${ }^{2}$, Chen-Hong Kuo ${ }^{1}$, Hung-Ju Lin ${ }^{2}$, Pi-Hua Liu ${ }^{3}$, Pei-Rony Chen ${ }^{4}$, Hsiu-Ching Hsu², \\ Bai-Chin Lee ${ }^{2}$, Yuan-Teh Lee ${ }^{2,5}$, Ming-Fong Chen ${ }^{2^{*}}$
}

\begin{abstract}
Background: Evidence of predictive power of various fatty acids on the risk of metabolic syndrome was scanty. We evaluated the role of various fatty acids, including saturated fat, monounsaturated fat, transfat, n- 6 fatty acid, eicosapentaenoic acid (EPA) and docosahexaenoic acid (DHA), for the risk of the metabolic syndrome in Taiwan.

Results: A nested case-control study based on 1000 cases of metabolic syndrome and 1:1 matched control subjects. For saturated fat, monounsaturated fat and transfat, the higher the concentration the higher the risk for metabolic syndrome: participants in the highest quintile had a 2.22-fold (95\% confidence interval [CI], 1.66 to 2.97) higher risk of metabolic syndrome. In addition, the participants in higher EPA quintiles were less likely to have the risk of metabolic syndrome (adjusted risk, 0.46 [0.34 to 0.61] for the fifth quintile). Participants in the highest risk group (low EPA and high transfat) had a 2.36-fold higher risk of metabolic syndrome (95\% Cl, 1.38 to 4.03), compared with those in the lowest risk group (high EPA and low transfat). For prediction power, the area under ROC curves increased from 0.926 in the baseline model to 0.928 after adding fatty acids. The net reclassification improvement for metabolic syndrome risk was substantial for saturated fat $(2.1 \%, P=0.05)$.
\end{abstract}

Conclusions: Plasma fatty acid components improved the prediction of the metabolic syndrome risk in Taiwan.

\section{Introduction}

Identifying dietary factors for the development of type 2 diabetes and metabolic syndrome is essential for primary prevention [1,2]. Dietary intake habits of fatty acids, including consumption of foods with high saturated fat and high transfat contents, are associated with insulin resistance and hyperlipidemia [3]. In addition, transfat has frequently been reported to be risk factors for cardiovascular diseases $[4,5]$, and the evidence for monounsaturated fats was inconclusive [6]. In contrast, marine-derived fatty acids, such as eicosapentaenoic acid (EPA) and docosahexaenoic acid (DHA) are inversely related to type 2 diabetes due to reduced inflammation and increased insulin sensitivity [7]. However, although there have been many studies about the association of specific fatty acids and the risk of cardiovascular diseases, there have only been a limited number of integrated comparison studies. Previous studies on fatty

\footnotetext{
* Correspondence: mfchen@ntu.edu.tw

${ }^{2}$ Department of Internal Medicine, National Taiwan University Hospital,

Taipei, Taiwan

Full list of author information is available at the end of the article
}

acids have focused on specific fatty acids only, without considering all of the fatty acids together [8-10]. In addition, the performance measures of each fatty acid have not been evaluated extensively, and clinical situations not considered simultaneously. Therefore, the aim of this study was to evaluate the specific role of various fatty acids, including saturated fat, monounsaturated fat, transfat, n-6 fatty acid, EPA and DHA, for the risk of the metabolic syndrome among ethnic Chinese adults in Taiwan. In addition, we evaluated the performance measures of these fatty acids and investigated the joint effect of two fatty acids together to test the prediction for the risk of metabolic syndrome.

\section{Results}

Table 1 presents the clinical, biochemical distribution, and fatty acid profiles of the study participants, specified by metabolic syndrome status. Compared with the control subjects, the case participants with metabolic syndrome were more likely to have a higher blood pressure, body mass index, waist circumference, total and LDL cholesterol, triglycerides, fasting glucose, uric acid,
C Biomed Central

다 2011 Chien et al; licensee BioMed Central Ltd. This is an Open Access article distributed under the terms of the Creative Commons Attribution License (http://creativecommons.org/licenses/by/2.0), which permits unrestricted use, distribution, and reproduction in any medium, provided the original work is properly cited. 
Table 1 Basic characteristics and fatty acid distributions of the study participants, specified by metabolic syndrome status

\begin{tabular}{|c|c|c|c|c|c|}
\hline & \multicolumn{2}{|c|}{ No metabolic syndrome $n=986$} & \multicolumn{3}{|c|}{ Metabolic syndrome, $\mathrm{n}=1000$} \\
\hline & Freq & $\%$ & Freq & $\%$ & \\
\hline Gender & & & & & 0.67 \\
\hline Women & 348 & 35.3 & 362 & 36.2 & \\
\hline Men & 638 & 64.7 & 638 & 63.8 & \\
\hline Smoking & 139 & 14.1 & 185 & 18.5 & 0.008 \\
\hline Drinking & 575 & 58.3 & 571 & 57.1 & 0.58 \\
\hline \multirow[t]{2}{*}{ Exercise } & 549 & 55.7 & 502 & 50.2 & 0.014 \\
\hline & Mean & Std Dev & Mean & Std Dev & $P$ \\
\hline Age, yr & 54.4 & 10.8 & 54.9 & 10.7 & 0.32 \\
\hline Systolic blood pressure, $\mathrm{mmHg}$ & 122.7 & 14.8 & 133.2 & 14.8 & $<.0001$ \\
\hline Diastolic blood pressure, $\mathrm{mmHg}$ & 72.9 & 9.8 & 79.2 & 10.2 & $<.0001$ \\
\hline Body mass index, $\mathrm{kg} / \mathrm{m}^{2}$ & 22.8 & 2.3 & 27.1 & 3.0 & $<.0001$ \\
\hline Waist, $\mathrm{cm}$ & 79.2 & 6.4 & 94.3 & 7.0 & $<.0001$ \\
\hline Total cholesterol, mg/dL & 202.7 & 36.4 & 206.8 & 36.8 & 0.012 \\
\hline Triglycerides, mg/dL & 115.4 & 74.0 & 178.3 & 104.5 & $<.0001$ \\
\hline $\mathrm{HDL}$ cholesterol, mg/dL & 43.1 & 9.7 & 38.5 & 7.0 & $<.0001$ \\
\hline LDL cholesterol, mg/dL & 116.3 & 31.5 & 124.9 & 32.7 & $<.0001$ \\
\hline Uric acid, mg/dL & 6.2 & 1.5 & 6.6 & 1.6 & $<.0001$ \\
\hline Fasting glucose, mg/dL & 93.1 & 22.2 & 105.0 & 28.1 & $<.0001$ \\
\hline \multicolumn{6}{|l|}{ Fatty acid, mg/dL } \\
\hline Saturated fat & 1713.0 & 512.9 & 1982.9 & 682.8 & $<.0001$ \\
\hline MUFA & 616.3 & 246.5 & 728.9 & 285.9 & $<.0001$ \\
\hline Trans fatty acid & 267.4 & 106.7 & 322.3 & 158.9 & $<.0001$ \\
\hline PUFA & 1488.2 & 347.9 & 1542.7 & 361.7 & 0.0006 \\
\hline N-6 fatty acids & 1297.4 & 313.4 & 1350.0 & 325.1 & 0.0003 \\
\hline N-3 fatty acids & 190.8 & 60.8 & 192.7 & 58.9 & 0.48 \\
\hline Marine fatty acids & 132.7 & 52.4 & 131.6 & 50.3 & 0.64 \\
\hline EPA & 21.5 & 14.5 & 20.3 & 12.5 & 0.06 \\
\hline DHA & 111.2 & 42.7 & 111.3 & 41.9 & 0.97 \\
\hline Total fat amount & 40.8 & 10.7 & 45.8 & 13.2 & $<.0001$ \\
\hline \multicolumn{6}{|l|}{ Fatty acid, $\%$ total fatty acids } \\
\hline Saturated fat & 41.8 & 4.4 & 43.0 & 4.6 & $<.0001$ \\
\hline MUFA & 14.8 & 2.6 & 15.7 & 2.6 & $<.0001$ \\
\hline Trans fatty acid & 6.52 & 1.84 & 6.95 & 2.00 & $<.0001$ \\
\hline PUFA & 36.8 & 4.7 & 34.4 & 4.8 & $<.0001$ \\
\hline N-6 fatty acids & 32.1 & 4.2 & 30.0 & 4.2 & $<.0001$ \\
\hline N-3 fatty acids & 4.76 & 1.37 & 4.34 & 1.21 & $<.0001$ \\
\hline Marine fatty acids & 3.32 & 1.23 & 2.97 & 1.09 & $<.0001$ \\
\hline EPA & 0.54 & 0.37 & 0.46 & 0.28 & $<.0001$ \\
\hline $\mathrm{DHA}$ & 2.78 & 0.99 & 2.51 & 0.91 & $<.0001$ \\
\hline
\end{tabular}

Abbreviations: MUFA: monounsaturated fatty acid; PUFA: polyunsaturated fatty acid; EPA: eicosapentaenoic acid; DHA: docosahexaenoic acid.

glomerular filtration rate, and a lower HDL cholesterol level. The case patients were more likely to smoke and less likely to exercise regularly. Due to the matched factors, age and gender were similar between the two groups. With regards to the absolute amount of fatty acids, participants with metabolic syndrome were more likely to have higher levels of saturated fatty acid, n-6 fatty acid, polyunsaturated fatty acid, and transfat.
However, after adjusting for total fatty acid intake, the case participants with metabolic syndrome tended to have higher levels of saturated fat, monounsaturated fatty acid, and lower n- 6 fatty acid, n-3 fatty acid, marine fatty acid, polyunsaturated fatty acid, and EPA and DHA concentrations compared with the control subjects. The age and gender adjusted Spearman correlation coefficients between the biochemical and fatty acid 
concentrations are shown in Additional file 1 Table S1. The correlations for absolute amount and relative percentage of fatty acids were different: for the absolute amount of fatty acids, most coefficients were positive for triglycerides, ranging from 0.11 for marine fatty acid to 0.74 . However, the coefficients changed modestly for total fat percentage: marine fatty acid, EPA and DHA were inversely related to waist circumference (ranging from -0.10 to -0.16$)$ and triglycerides $(-0.24$ to -0.29$)$, and positively related to HDL cholesterol (0.16 to 0.21 ). Other clinical variables, including blood pressure, total cholesterol, LDL cholesterol, uric acid, fasting glucose, and glomerular filtration rate, were not significantly associated with EPA and DHA.

According to the quintiles of various fatty acids, including saturated fat, monounsaturated fat, transfat, n-6 fatty acid, and EPA and DHA concentrations in the control subjects, we examined the adjusted odds ratios and $95 \%$ confidence intervals $(\mathrm{CI})$, and tested for trend for the risk of metabolic syndrome (Table 2). For saturated fat, monounsaturated fat and transfat, the higher the concentration, the higher the risk for the metabolic syndrome. Compared with those in lower saturated fat quintiles, participants in the highest quintile had a 2.22fold increased risk (95\% confidence interval [CI], 1.66 to 2.97, $P<0.0001$ ) of having the metabolic syndrome. The effects persisted after multiple adjustments. Similar positive associations existed for monounsaturated fat and transfat: the adjusted risks in the participants in the fifth quintile were 1.89 (95\% CI, 1.18 to 3.01 ) for monounsaturated fat and 1.53 (95\% CI, 0.99 to 2.35, test for trend, $P=0.002$ ) for transfat. However, an inverse association was found for n-6 fatty acid, EPA and DHA. Compared with those in the lowest EPA quintile, the participants in higher EPA quintiles were less likely to have a risk of metabolic syndrome (age and gender adjusted risk, 0.62 [ $95 \% \mathrm{CI}, 0.47$ to 0.81 ] for the second, 0.60 [0.46 to 0.79 ] for the third, 0.60 [0.45 to 0.78$]$ for the fourth, and 0.46 [0.34 to 0.61$]$ for the fifth quintile, test for trend, $P<0.0001)$. The protective effects of EPA were still persistent after multiple adjustments. Similar patterns were found for DHA levels. Compared with the first DHA quintile, the multivariate adjusted risks for consequent quintiles were 0.87 (0.58 to 1.29$), 0.65$ (0.43 to 1.00$), 0.51$ (0.33 to 0.78 ) and $0.54(0.35$ to 0.84$)$, respectively. The estimated risk for continuous variables, specified by concentration as a unit or by one standard deviation unit, showed various standardized coefficients, the absolute values ranging from 0.15 for saturated fat to 0.29 for $\mathrm{n}-6$ fatty acid (Additional file 1 Table S2).

To test the performance measures of different fatty acid profiles, we estimated the area under the receiver operative characteristics (ROC) curves (AUCs) in the baseline model, and tested the incremental values after adding one fatty acid (Table 3). If treated as quintile values, the AUC increased from 0.926 in the baseline model to 0.928 in the additional $\mathrm{n}-6$ fatty acid model $(P$ for difference among 7 models, 0.009). The reclassification tables with and without EPA and transfat are shown in Additional file 1 Table S3, and the performance measure values are listed in Table 4 . The net reclassification improvement was substantial for saturated fat $(2.1 \%, 95 \% \mathrm{CI}, 0.0 \%$ to $4.2 \%, P=0.05)$, and the integrated discriminative improvement values for fatty acids were significant, ranging from $0.2 \%$ for saturated fat and DHA to $0.7 \%$ for monounsaturated fat and n-6 fatty acid (all $P<0.05$ ).

In mutually adjusted models for two fatty acids, most fatty acids remained significant and strong risk factors for metabolic syndrome (Table 5). For example, the adjusted odds ratio of the highest EPA quintile was 0.55 ( $95 \%$ CI, 0.35 to 0.86 ) and that of the highest saturated fat quintile 1.56 (95\% CI, 1.00 to 2.45). In joint analyses, participants in the highest risk group (low EPA and high transfat) had a 2.4-fold higher risk of metabolic syndrome (adjusted odds ratio, 2.36, 95\% CI, 1.38 to 4.03, $\mathrm{P}=0.002$ ), compared with those in the lowest risk group (high EPA and low transfat) (Figure 1).

\section{Discussion}

Our study clearly demonstrated that plasma fatty acid components improved the prediction of the risk of the metabolic syndrome among ethnic Chinese adults in Taiwan. Significant simultaneous effects and joint additive effects were also shown for these fatty acids. Additional information on fatty acids is necessary to predict the status of metabolic syndrome in adult populations, implied by a good performance measure. Extrapolations to the clinical purpose of these findings were feasible for management of metabolic syndrome in primary prevention setting. Because of high prevalence of metabolic syndrome worldwide, our findings would be applied in clinical setting.

Our findings were consistent with previous studies. Both $n-6$ and $n-3$ polyunsaturated fatty acids have been associated with lower cardiovascular risk $[11,12]$. Dietary fat quality has been reported to influence the activity of enzymes involved in the desaturation of fatty acids in the body, and data from metabolic and clinical studies support recommendations to replace saturated and trans fat with unsaturated fat in the prevention of cardiovascular disease. Our data support that saturated fat, monounsaturated fat, and transfat are significantly associated with the risk of metabolic syndrome. For the protective role of fatty acids, marine fatty acids, especially EPA and DHA, have been shown to play a protective role in the risk of metabolic syndrome $[12,13]$. 
Table 2 The numbers of the case control subjects according to fatty acid quintiles and adjusted odds ratios in the study participants

\begin{tabular}{|c|c|c|c|c|c|c|c|c|c|c|c|c|c|c|}
\hline & Q1 & Q2 & & & Q3 & & & Q4 & & & Q5 & & & $P$, Trend \\
\hline \multicolumn{15}{|c|}{ Saturated fat, \% total fatty acids } \\
\hline Range & $<38.0$ & $38.0-40.1$ & & & $40.1-42.5$ & & & $42.5-45.7$ & & & $>=45.7$ & & & \\
\hline Median & 36.6 & 39.2 & & & 41.2 & & & 44.1 & & & 48.3 & & & \\
\hline Control & 195 & 196 & & & 196 & & & 197 & & & 195 & & & $<.0001$ \\
\hline Cases & 127 & 168 & & & 221 & & & 201 & & & 281 & & & \\
\hline \multicolumn{2}{|l|}{ Odds ratio } & OR & \multicolumn{2}{|c|}{$95 \% \mathrm{Cl}$} & OR & \multicolumn{2}{|c|}{$95 \% \mathrm{Cl}$} & OR & \multicolumn{2}{|c|}{$95 \% \mathrm{Cl}$} & OR & \multicolumn{2}{|c|}{$95 \% \mathrm{Cl}$} & \\
\hline Model 1 & 1 & 1.31 & 0.97 & 1.78 & 1.75 & 1.30 & 2.34 & 1.57 & 1.16 & 2.11 & 2.22 & 1.66 & 2.97 & $<.0001$ \\
\hline Model 2 & 1 & 1.05 & 0.67 & 1.64 & 1.48 & 0.97 & 2.28 & 1.20 & 0.78 & 1.84 & 1.94 & 1.27 & 2.96 & 0.002 \\
\hline Model 3 & 1 & 1.03 & 0.65 & 1.63 & 1.33 & 0.85 & 2.06 & 1.03 & 0.66 & 1.60 & 1.68 & 1.08 & 2.62 & 0.025 \\
\hline \multicolumn{15}{|c|}{ MUFA, $\%$ total fatty acids } \\
\hline Range & $<12.6$ & $12.6-14.1$ & & & $14.1-15.4$ & & & $15.4-16.9$ & & & $>=16.9$ & & & \\
\hline Median & 11.5 & 13.5 & & & 14.7 & & & 16.2 & & & 18.2 & & & \\
\hline Control & 195 & 196 & & & 197 & & & 196 & & & 195 & & & $<.0001$ \\
\hline Cases & 117 & 150 & & & 161 & & & 256 & & & 314 & & & \\
\hline Model 1 & 1 & 1.27 & 0.93 & 1.74 & 1.38 & 1.01 & 1.89 & 2.22 & 1.65 & 2.99 & 2.76 & 2.06 & 3.70 & $<.0001$ \\
\hline Model 2 & 1 & 1.26 & 0.80 & 1.99 & 1.01 & 0.64 & 1.59 & 1.89 & 1.22 & 2.91 & 2.12 & 1.37 & 3.26 & $<.0001$ \\
\hline Model 3 & 1 & 1.14 & 0.71 & 1.83 & 0.94 & 0.59 & 1.51 & 1.78 & 1.14 & 2.80 & 1.89 & 1.18 & 3.01 & 0.001 \\
\hline \multicolumn{15}{|c|}{ Transfat, \% total fatty acids } \\
\hline Range & $<5.2$ & $5.2-5.9$ & & & $5.9-6.7$ & & & $6.7-7.7$ & & & $>=7.7$ & & & \\
\hline Median & 4.6 & 5.5 & & & 6.3 & & & 7.2 & & & 8.7 & & & \\
\hline Control & 195 & 197 & & & 194 & & & 197 & & & 196 & & & $<.0001$ \\
\hline Cases & 147 & 147 & & & 178 & & & 222 & & & 303 & & & \\
\hline Model 1 & 1 & 0.99 & 0.73 & 1.34 & 1.23 & 0.91 & 1.65 & 1.50 & 1.12 & 2.00 & 2.05 & 1.55 & 2.72 & $<.0001$ \\
\hline Model 2 & 1 & 0.88 & 0.56 & 1.37 & 1.18 & 0.77 & 1.83 & 1.81 & 1.20 & 2.75 & 1.68 & 1.11 & 2.53 & 0.0003 \\
\hline Model 3 & 1 & 0.81 & 0.51 & 1.29 & 1.09 & 0.69 & 1.72 & 1.65 & 1.07 & 2.54 & 1.53 & 0.99 & 2.35 & 0.002 \\
\hline \multicolumn{15}{|c|}{ N-6 fatty acids, $\%$ total fatty acids } \\
\hline Range & $<28.7$ & 28.7-31.1 & & & $31.1-33.3$ & & & 33.3-35.8 & & & $>=35.8$ & & & \\
\hline Median & 26.4 & 29.9 & & & 32.2 & & & 34.4 & & & 37.4 & & & \\
\hline Control & 195 & 196 & & & 196 & & & 196 & & & 196 & & & $<.0001$ \\
\hline Cases & 363 & 225 & & & 181 & & & 159 & & & 70 & & & \\
\hline Model 1 & 1 & 0.62 & 0.48 & 0.80 & 0.50 & 0.38 & 0.65 & 0.43 & 0.33 & 0.57 & 0.19 & 0.14 & 0.27 & $<.0001$ \\
\hline Model 2 & 1 & 0.83 & 0.57 & 1.21 & 0.65 & 0.44 & 0.96 & 0.55 & 0.38 & 0.82 & 0.31 & 0.20 & 0.49 & $<.0001$ \\
\hline Model 3 & 1 & 0.84 & 0.57 & 1.24 & 0.69 & 0.46 & 1.04 & 0.64 & 0.42 & 0.97 & 0.36 & 0.22 & 0.59 & $<.0001$ \\
\hline \multicolumn{8}{|c|}{ EPA, \% total fatty acids } & & & & & & & $<.0001$ \\
\hline Range & $<0.28$ & $0.28-0.38$ & & & $0.38-0.52$ & & & $0.52-0.76$ & & & $>=0.76$ & & & \\
\hline Median & 0.22 & 0.33 & & & 0.44 & & & 0.62 & & & 0.94 & & & \\
\hline Control & 196 & 196 & & & 196 & & & 196 & & & 195 & & & \\
\hline Cases & 299 & 186 & & & 185 & & & 186 & & & 142 & & & \\
\hline Model 1 & 1 & 0.62 & 0.47 & 0.81 & 0.60 & 0.46 & 0.79 & 0.60 & 0.45 & 0.78 & 0.46 & 0.34 & 0.61 & $<.0001$ \\
\hline Model 2 & 1 & 0.61 & 0.41 & 0.92 & 0.51 & 0.34 & 0.76 & 0.67 & 0.45 & 0.99 & 0.51 & 0.33 & 0.77 & 0.013 \\
\hline Model 3 & 1 & 0.60 & 0.39 & 0.91 & 0.51 & 0.34 & 0.78 & 0.68 & 0.45 & 1.03 & 0.51 & 0.33 & 0.80 & 0.028 \\
\hline \multicolumn{15}{|c|}{ DHA, $\%$ total fatty acids } \\
\hline Range & $<1.97$ & $1.97-2.45$ & & & $2.45-2.89$ & & & $2.89-3.56$ & & & $>=3.56$ & & & \\
\hline Median & 1.6 & 2.2 & & & 2.7 & & & 3.2 & & & 4.0 & & & \\
\hline Control & 96 & 196 & & & 195 & & & 197 & & & 195 & & & \\
\hline Cases & 281 & 256 & & & 167 & & & 161 & & & 133 & & & \\
\hline Model 1 & 1 & 0.89 & 0.69 & 1.16 & 0.58 & 0.44 & 0.77 & 0.55 & 0.42 & 0.73 & 0.45 & 0.34 & 0.60 & $<.0001$ \\
\hline Model 2 & 1 & 0.97 & 0.66 & 1.43 & 0.68 & 0.45 & 1.02 & 0.54 & 0.36 & 0.82 & 0.58 & 0.38 & 0.89 & 0.001 \\
\hline Model 3 & 1 & 0.90 & 0.60 & 1.34 & 0.69 & 0.45 & 1.05 & 0.55 & 0.35 & 0.85 & 0.59 & 0.38 & 0.92 & 0.003 \\
\hline
\end{tabular}

Model 1: adjusted for age and gender.

Model 2: additional body mass index, smoking, drinking and exercise.

Model 3: additional LDL cholesterol, systolic and diastolic blood pressure, uric acid, fasting glucose levels and total fat amount in plasma.

Abbreviations see Table 1. 
Table 3 Area under the ROC curves comparison with the base model

\begin{tabular}{|c|c|c|c|c|c|c|}
\hline \multirow[b]{2}{*}{ Base model } & \multirow{2}{*}{$\begin{array}{l}\text { AUC } \\
0.926\end{array}$} & \multicolumn{2}{|c|}{$95 \% \mathrm{Cl}$} & \multirow[t]{2}{*}{ AUC } & \multicolumn{2}{|c|}{$95 \% \mathrm{Cl}$} \\
\hline & & 0.915 & 0.936 & & & \\
\hline \multicolumn{7}{|l|}{ Additional for: } \\
\hline & \multicolumn{3}{|c|}{ Continuous variables * } & \multicolumn{3}{|c|}{ Quintile variables ** } \\
\hline Saturated fat, \% fat & 0.926 & 0.915 & 0.937 & 0.927 & 0.916 & 0.937 \\
\hline MUFA, \% fat & 0.927 & 0.916 & 0.937 & 0.928 & 0.917 & 0.939 \\
\hline Transfat, \% fat & 0.926 & 0.915 & 0.937 & 0.927 & 0.917 & 0.938 \\
\hline N-6 fatty acids, \% fat & 0.927 & 0.916 & 0.938 & 0.928 & 0.917 & 0.938 \\
\hline EPA, \% fat & 0.926 & 0.916 & 0.937 & 0.927 & 0.916 & 0.938 \\
\hline $\mathrm{DHA}, \%$ fat & 0.926 & 0.916 & 0.937 & 0.926 & 0.915 & 0.937 \\
\hline
\end{tabular}

* test for 7 models, $p=0.08$.

** test for 7 models, $p=0.009$.

The base model included: age, gender, body mass index, smoking, drinking and exercise, LDL cholesterol, systolic and diastolic blood pressure, uric acid, fasting glucose levels and total fat amount in plasma.

AUC: area under the ROC curve; $\mathrm{Cl}$ : confidence interval.

Our approach, integrating all fatty acid measurements instead of just one specific fatty acid, extended the understanding of the role of fatty acids on the risk of metabolic syndrome. After considering other fatty acids, DHA and EPA still played an important role for reducing the risk of metabolic syndrome status. The inverse relationship between marine $\mathrm{n}-3$ fatty acids and metabolic syndrome may implicate the protective effect of n-3 PUFAs intake in isocaloric substitution for other

Table 4 Performance measures of the models including various fatty acid levels

\begin{tabular}{|c|c|c|c|c|c|c|c|c|}
\hline & $\begin{array}{l}\text { NRI, } \\
\%\end{array}$ & $\begin{array}{c}95 \% \\
\mathrm{Cl}\end{array}$ & & $P$ & $\begin{array}{l}\text { IDI, } \\
\%\end{array}$ & $\begin{array}{c}95 \% \\
\mathrm{Cl}\end{array}$ & & $P$ \\
\hline \multicolumn{9}{|l|}{ Quintile variables } \\
\hline $\begin{array}{l}\text { Saturated fat, \% } \\
\text { fat }\end{array}$ & 2.1 & 0.0 & 4.2 & 0.05 & 0.2 & 0.0 & 0.5 & 0.036 \\
\hline MUFA, \% fat & 2.2 & -0.4 & 4.8 & 0.09 & 0.7 & 0.3 & 1.0 & 0.0001 \\
\hline Transfat, \% fat & 1.7 & -0.8 & 4.2 & 0.17 & 0.5 & 0.2 & 0.9 & 0.001 \\
\hline $\begin{array}{l}\text { N-6 fatty acids, \% } \\
\text { fat }\end{array}$ & 1.5 & -0.9 & 3.9 & 0.22 & 0.7 & 0.3 & 1.0 & 0.0001 \\
\hline EPA, \% fat & 1.4 & -0.9 & 3.7 & 0.24 & 0.4 & 0.1 & 0.7 & 0.002 \\
\hline DHA, \% fat & 0.2 & -1.5 & 1.9 & 0.81 & 0.2 & 0.0 & 0.4 & 0.046 \\
\hline \multicolumn{9}{|c|}{ Continuous variables } \\
\hline $\begin{array}{l}\text { Saturated fat, \% } \\
\text { fat }\end{array}$ & 0.7 & -1.0 & 2.4 & 0.42 & 0.1 & -0.1 & 0.3 & 0.09 \\
\hline MUFA, \% fat & 1.4 & -0.5 & 3.3 & 0.15 & 0.3 & 0.1 & 0.5 & 0.002 \\
\hline Transfat, \% fat & 0.3 & -1.3 & 1.9 & 0.71 & 0.1 & -0.1 & 0.3 & 0.08 \\
\hline $\begin{array}{l}\text { N-6 fatty acids, \% } \\
\text { fat }\end{array}$ & 1.6 & -0.7 & 3.9 & 0.17 & 0.5 & 0.2 & 0.8 & 0.001 \\
\hline EPA, \% fat & 1.7 & -0.2 & 3.6 & 0.08 & 0.2 & 0.0 & 0.5 & 0.022 \\
\hline DHA, \% fat & 1.3 & -0.6 & 3.2 & 0.18 & 0.2 & 0.0 & 0.4 & 0.031 \\
\hline
\end{tabular}

NRI cutoff points: $0.1,0.5$, and 0.9 .

Abbreviations: MUFA: monounsaturated fatty acid; PUFA: polyunsaturated fatty acid; EPA: eicosapentaenoic acid; DHA: docosahexaenoic acid; NRI: net reclassification improvement; IDI: integrated discrimination improvement; $\mathrm{Cl}$, confidence interval. fats on metabolic syndrome, which was associated with the increased risk of cardiovascular diseases [14]. Observational studies and clinical trials have shown that intake of EPA and DHA was associated to lower the cardiovascular risk [11]. The effects of $n-3$ fatty acids metabolic syndrome on cardiovascular disease were mediated through reducing some intermediate markers, such as metabolic syndrome components and inflammatory biomarkers [15]. The protective roles of EPA and DHA for the risk of metabolic syndrome might provide the early prevention of cardiovascular events. Our study clearly demonstrated the additional beneficial effects of EPA and DHA for the risk of the metabolic syndrome.

\section{Study strength and limitations}

To the best of our knowledge, this study was one of the few studies to examine the cumulative effects of fatty acids on the risk of metabolic syndrome. The study sample size was relatively large and the characteristics of the study participants were homogeneous; so the results were internally consistent. However, several limitations should be mentioned. First, the fatty acids were only measured once, and variations due to the seasonal availability of foods were not evaluated. However, the nondifferential misclassification due to measurement would underestimate the true effect. We believe the true association between fatty acids and the risk of metabolic syndrome may be more significant. Second, we did not collect the dietary habits in this study. However, biochemical measurements of fatty acids have a reasonable validity with the information from food frequency questionnaires [16,17].

\section{Conclusion}

In conclusion, plasma fatty acid concentrations improved the prediction of the risk of metabolic syndrome. Additional joint effects were also found between two fatty acid levels. Among them, n- 6 fatty acid and marine derived n-3 fatty acids, i.e., EPA and DHA, were protective factors for the risk of metabolic syndrome. Further studies on the mechanism of various fatty acids on the pathway of metabolic syndrome are warranted.

\section{Methods}

\section{Study design and population}

The study design was a nested case-control study design based on 8,911 adult participants who were recruited from the Health Management Center of one tertiary hospital from January 2004 to December 2007. All participants provided written informed consent with the study protocol being reported elsewhere [18-20]. In brief, details of socioeconomic status, along with medical and medication histories were collected by questionnaires, and standardized clinical procedures were 
Table 5 Combination of two fatty acids adjusted confounding factor in multivariable adjusted models

\begin{tabular}{llccccccccc}
\hline & & \multicolumn{3}{c}{ Fatty Acid 1 } & & & \multicolumn{2}{c}{ Fatty Acid 2 } \\
Model & Marker & Odds ratio & $\mathbf{9 5 \%}$ Cl & P trend & Marker & Odds ratio & $\mathbf{9 5 \%}$ Cl & P trend \\
\hline 1 & EPA, \% fat & 0.55 & 0.35 & 0.86 & 0.039 & Saturated fat, \% fat & 1.56 & 1.00 & 2.45 & 0.035 \\
2 & EPA, \% fat & 0.49 & 0.31 & 0.76 & 0.027 & MUFA, \% fat & 2.07 & 1.29 & 3.32 & 0.001 \\
3 & EPA, \% fat & 0.52 & 0.33 & 0.81 & 0.057 & Transfat, \% fat & 1.38 & 0.89 & 2.14 & 0.004 \\
4 & EPA, \% fat & 0.48 & 0.31 & 0.76 & 0.012 & N-6 fatty acids, \% fat & 0.36 & 0.22 & 0.59 & $<.0001$ \\
5 & EPA, \% fat & 0.63 & 0.38 & 1.06 & 0.43 & DHA, \% fat & 0.72 & 0.42 & 1.21 & 0.033 \\
6 & DHA, \% fat & 0.65 & 0.39 & 1.09 & 0.036 & Saturated fat, \% fat & 1.28 & 0.77 & 2.12 & 0.41 \\
7 & DHA, \% fat & 0.37 & 0.23 & 0.61 & $<.0001$ & MUFA, \% fat & 3.01 & 1.81 & 5.02 & $<.0001$ \\
8 & DHA, \% fat & 0.72 & 0.43 & 1.19 & 0.10 & Transfat, \% fat & 1.23 & 0.75 & 2.01 & 0.06 \\
9 & DHA, \% fat & 0.73 & 0.45 & 1.16 & 0.076 & N-6 fatty acids, \% fat & 0.41 & 0.25 & 0.69 & 0.001 \\
10 & N-6 fatty acids, \% fat & 0.22 & 0.11 & 0.46 & 0.000 & Saturated fat, \% fat & 0.60 & 0.30 & 1.17 & 0.17 \\
11 & N-6 fatty acids, \% fat & 0.24 & 0.14 & 0.41 & $<.0001$ & MUFA, \% fat & 3.02 & 1.82 & 5.02 & $<.0001$ \\
12 & N-6 fatty acids, \% fat & 0.33 & 0.16 & 0.68 & 0.008 & Transfat, \% fat & 0.78 & 0.41 & 1.50 & 0.88 \\
13 & Transfat, \% fat & 1.63 & 0.90 & 2.92 & 0.032 & Saturated fat, \% fat & 1.00 & 0.54 & 1.84 & 0.99 \\
14 & Transfat, \% fat & 2.98 & 1.82 & 4.90 & $<.0001$ & MUFA, \% fat & 4.11 & 2.37 & 7.11 & $<.0001$ \\
15 & MUFA, \% fat & 9.25 & 4.68 & 18.3 & $<.0001$ & Saturated fat, \% fat & 7.77 & 4.14 & 14.6 & $<.0001$ \\
\hline
\end{tabular}

Abbreviations as for Table 1.

Results are from separate models of each combination of fatty acid profiles. Odds ratios and 95\% confidence intervals in the highest compared with the lowest quintile of each fatty acid. P for trend across quintiles of each profile, and each fatty acid profile was added to the model in quintile with 4 dummy variables. Adjusted for age, gender, BMI, smoking, drinking and exercise, LDL cholesterol, systolic and diastolic blood pressure, uric acid, fasting glucose levels and total fat amount in plasma.

undertaken. We excluded the participants with concurrent severe medical diseases such as cancer and heart failure. The participants signed informed consent forms, and the protocol was approved by the Institutional Research Board of the hospital.

Details of the subjects' medical histories such as medication, hospitalization and smoking status were included in the structural questionnaires. Standardized procedures for the physical examination, such as anthropometric

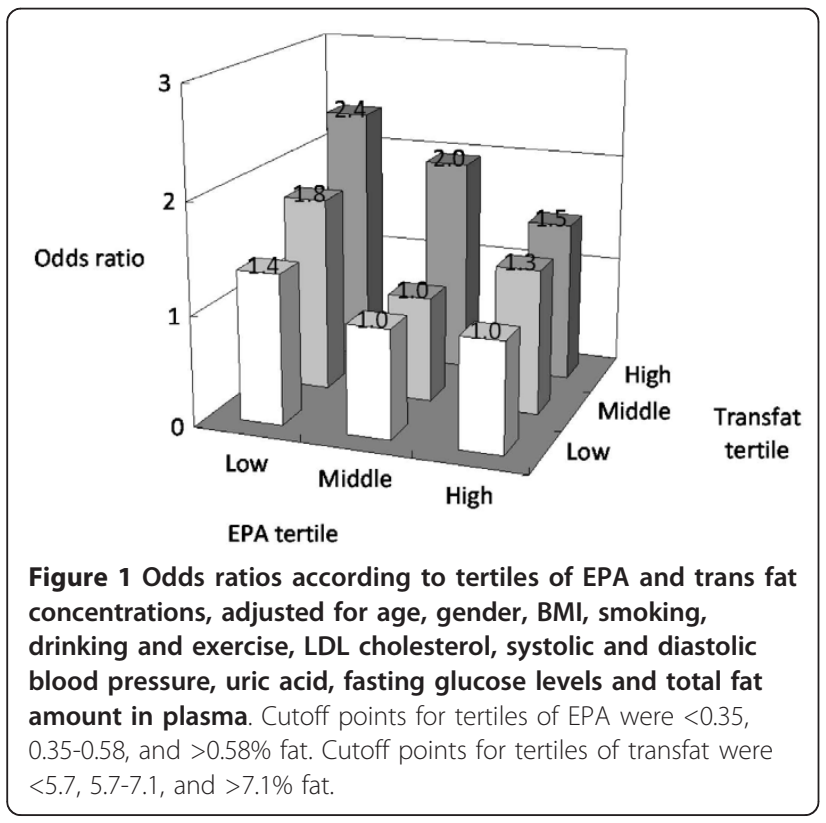

measures and blood pressure, were performed $[18,19]$. Blood pressure was measured in a resting position by trained medical assistants. We defined the lifestyle and clinical covariates as smoking (current smoker, ever-smoker and never smoker), alcohol intake (regular/no), occupation (business and service work), regular exercise (yes/ no), family history of coronary heart disease (yes/no), baseline hypertension (yes/no, defined by a blood pressure of at least $140 / 90 \mathrm{~mm} \mathrm{Hg}$ or on medication), and diabetes mellitus (yes/no, defined by a fasting plasma glucose of at least $126 \mathrm{mg} / \mathrm{dl}$ or on medication).

\section{Blood sampling and analytic methods}

The procedures for blood sampling and analytic methods have been described in previous studies [18,21]. In brief, blood samples were collected from each participant after fasting for at least 12 hours. Serum total cholesterol levels were measured using the CHOD-PAP method (Boehringer Mannheim, Germany). HDL-C was measured following precipitation of apolipoprotein B-containing lipoproteins with phosphotungstic acid and magnesium ions (Boehringer Mannheim, Germany). Triglyceride concentrations were measured by the GPODAOS method (Wako Co., Japan). All of the lipids were measured using a Hitachi 7450 automated analyzer (Hitachi, Japan). LDL-C concentrations were calculated using the Friedewald formula. Plasma fasting glucose and 2-hr postprandial glucose concentrations were measured using a Hitachi 7450 automated analyzer (Hitachi, Japan). HbA1c levels were measured using a DCA 2000 
analyzer (Bayer Diagnostics, Elkhart, IN). All of the measurements were carried out in a single hospital. The coefficient of variation was $5 \%$.

\section{Case ascertainment and matched control selection}

Metabolic syndrome was defined according to the updated criteria of the Adult Treatment Panel III [22] and International Diabetes Federation (IDF), which have been shown to have excellent consistency [18]. We used the IDF criteria to ascertain the metabolic syndrome cases as those who had a major component of central obesity (defined by a waist circumference greater than $90 \mathrm{~cm}$ in men and $80 \mathrm{~cm}$ in women) and two other components from the following: 1) blood pressure of at least 130/85 $\mathrm{mmHg}$ or undergoing treatment for hypertension; 2) serum triglycerides of at least $1.7 \mathrm{mmol} / \mathrm{L}$ (150 mg/dL); 3) HDL cholesterol less than $1.0 \mathrm{mmol} / \mathrm{L}$ $(40 \mathrm{mg} / \mathrm{dL})$ in men and less than $1.3 \mathrm{mmol} / \mathrm{L}(50 \mathrm{mg} /$ $\mathrm{dL}$ ) in women; and 4) fasting glucose $>=5.5 \mathrm{mmol} / \mathrm{L}$ $(100 \mathrm{mg} / \mathrm{dL})$. In all, 1869 individuals (21\%) were classified as having metabolic syndrome. Next, we randomly selected 1000 of these individuals and then control subjects were frequency-matched according to age and gender.

Fatty acid profile measurements in gas chromatography A 10-ml tube of EDTA-anticoagulated blood was collected, refrigerated at the site centers, and sent back within 3 hours to the National Taiwan University Hospital core laboratory. The blood was centrifuged at 800 $\times g$ for $10 \mathrm{~min}$, then plasma was separated and dispensed into several aliquots and frozen at $-70^{\circ} \mathrm{C}$ for analysis for fatty acid content by a single technician. After thawing, $0.5 \mathrm{~mL}$ of plasma was extracted with $0.5 \mathrm{~mL}$ methanol followed by $1.0 \mathrm{~mL}$ chloroform under a nitrogen atmosphere, and the lipid extract was filtered to remove protein. The methyl esters were then separated and measured on a 5890 gas chromatograph (Hewlett Packard, Avondale, PA) equipped with a $30 \mathrm{~m}$-FFAT WCOT glass capillary column (J \& W Scientific, Folsom, CA) and a flame-ionization detector. The identities of 29 individual fatty acid peaks were ascertained by comparing each peak's retention time relative to the retention times of FAs of synthetic standards of known FA components. The relative amount of each FA (\% of total FAs) was quantified by integrating the area under the peak, and dividing the result by the total area for all FAs. To minimize transcription errors, the data from the gas chromatogram was electronically transferred to a computer for data analysis.

\section{Statistical analysis}

All data were presented as mean and standard deviation for continuous variables and contingency tables for categorical data, and were listed by status of case patients and control subjects. The age and gender adjusted Spearman correlation coefficients were examined. To compare different fatty acids in predicting the risk of metabolic syndrome, we used the following strategies:

First, the frequency of each fatty acid in cases and controls was calculated and stratified by fatty acid quintiles. We analyzed the association between various fatty acids and the risk of metabolic syndrome using the logistic regression model, adjusting for potential confounding factors. We specified three logistic models to evaluate the adjusted odds ratios of quintile values. Model 1 was adjusted for age (continuous variable) and gender only. Model 2 included additional body mass index (continuous variable), smoking, alcohol intake and exercise status. Model 3 included additional continuous LDL cholesterol, systolic and diastolic blood pressure, uric acid, fasting glucose levels and total fat amount in plasma. To test for linear trends across fatty acid categories, we used the median fatty acid profile levels within quintiles as a continuous variable. We also tested the goodness of fit of the model using the Hosmer and Lemeshow test [23], and the model fit was acceptable.

Second, we compared the performance of the models with and without fatty acid measurements using the area under the receiver operating characteristic curve (AUC). The curve is a graph of sensitivity versus 1-specificity (or false-positive rate) for various cutoff definitions of a positive diagnostic test result [24]. Statistical differences in the AUCs were compared using the method of DeLong et al. [25], and the difference of AUC was used to compare the discriminatory capability among the models.

Third, we provided several additional statistics, including integrated discrimination improvement (IDI) and net reclassification improvement (NRI) [26] for the comparison of nested models with and without fatty acids, because the AUC value is not the best discriminatory statistic for prediction power [26-28]. In brief, NRI was based on the reclassification tables and was calculated from a sum of differences between the 'upward' movement in categories for event subjects and the "downward' movement in those for nonevent subjects [26]. A priori risk categories were defined according to the a priori risk categories of stroke $(0-10 \%, 10-50 \%, 50-90 \%$, and $>=$ $90 \%$ ), and the reclassification tables according to the metabolic syndrome were specified. The IDI is considered to be the difference between improvement in average sensitivity and any potential increase in average 'one minus specificity' [26], and is estimated as the difference in Yates discrimination slopes between the nested models $[29,30]$. The reclassification table as a tool for comparing models was suggested by Ridker and colleagues [28]. 
All statistical analyses were performed using SAS version 9.1 (SAS Institute, Inc., Cary, NC) and STATA version 9.1 (Stata Corp., College Station, Texas).

\section{Additional material}

Additional file 1: Additional Tables S1-S3. Including supplementary tables for Spearman partial correlation coefficients, Adjusted odds ratios and $95 \%$ confidence intervals for fatty acid concentrations, specified by continuous variables, and Net reclassification tables.

\section{Acknowledgements}

The study was partly supported by grants from the National Taiwan University Hospital and National Science Council in Taiwan (98-S1056, NSC 97-2314-B-002 -130 -MY3, 97-3112-B-002-034-). The authors express thanks to the participants in this study.

\section{Author details}

'Institute of Epidemiology \& Preventive Medicine, College of Public School, National Taiwan University, Taipei, Taiwan. ${ }^{2}$ Department of Internal Medicine, National Taiwan University Hospital, Taipei, Taiwan. ${ }^{3}$ School of Medicine, Chang-Gun University, Lin-Kou, Taoyuan, Taiwan. ${ }^{4}$ Department of Nutrition, National Taiwan University Hospital, Taipei, Taiwan. ${ }^{5}$ Chinese Medical University Hospital, Taichung, Taiwan

\section{Authors' contributions}

$\mathrm{KLC}, \mathrm{YTL}$ and MFC proposed the study design. $\mathrm{KLC}, \mathrm{CLC}, \mathrm{CHK}, \mathrm{HJL}$, and $\mathrm{BCL}$ participated in data collection. KLC and PHL performed statistical and genetic analysis. PRC provided consultation and modified the draft. $\mathrm{HCH}$ performed fatty acid measurements and quality control. YTL and MFC conceived of the study, and participated in its design and coordination and helped to draft the manuscript. All authors read and approved the final manuscript.

\section{Competing interests}

The authors declare that they have no competing interests.

Received: 28 December 2010 Accepted: 21 February 2011

Published: 21 February 2011

\section{References}

1. Siscovick DS, Lemaitre RN, Mozaffarian D: The fish story: a diet-heart hypothesis with clinical implications: $\mathrm{n}-3$ polyunsaturated fatty acids, myocardial vulnerability, and sudden death. Circulation 2003, 107(21):2632-2634

2. Mensink RP, Katan MB: Effect of dietary fatty acids on serum lipids and lipoproteins. A meta-analysis of 27 trials. Arterioscler Thromb 1992. 12(8):911-919.

3. Mensink R: Effects of the individual saturated fatty acids on serum lipids and lipoprotein concentrations. Am J Clin Nutr 1993, 57(5):711S-714.

4. Merchant AT, Kelemen LE, de Koning L, Lonn E, Vuksan V, Jacobs R, Davis B, Teo KK, Yusuf S, Anand SS: Interrelation of saturated fat, trans fat, alcohol intake, and subclinical atherosclerosis. Am J Clin Nutr 2008, 87(1):168-174.

5. Micha R, Mozaffarian D: Trans fatty acids: effects on cardiometabolic health and implications for policy. Prostaglandins Leukot Essent Fatty Acids 2008, 79(3-5):147-152.

6. Hu FB, Stampfer MJ, Manson JE, Rimm E, Colditz GA, Rosner BA, Hennekens CH. Willett WC: Dietary Fat Intake and the Risk of Coronary Heart Disease in Women. New England Journal of Medicine 1997, 337(21):1491-1499.

7. von Schacky C: Omega-3 fatty acids and cardiovascular disease. Curr Opin Clin Nutr Metab Care 2007, 10(2):129-135.

8. Motoyama KR, Curb JD, Kadowaki T, El-Saed A, Abbott RD, Okamura T, Evans RW, Nakamura Y, Sutton-Tyrrell K, Rodriquez BL, et al: Association of serum $n-6$ and $n-3$ polyunsaturated fatty acids with lipids in 3 populations of middle-aged men. Am J Clin Nutr 2009, 90(1):49-55
9. Yamagishi K, Iso H, Date C, Fukui M, Wakai K, Kikuchi S, Inaba Y, Tanabe N Tamakoshi A: Fish, omega-3 polyunsaturated fatty acids, and mortality from cardiovascular diseases in a nationwide community-based cohort of Japanese men and women the JACC (Japan Collaborative Cohort Study for Evaluation of Cancer Risk) Study. J Am Coll Cardiol 2008, 52(12):988-996.

10. Sekikawa A, Curb JD, Ueshima H, El-Saed A, Kadowaki T, Abbott RD, Evans RW, Rodriguez BL, Okamura T, Sutton-Tyrrell K, et al: Marinederived $\mathrm{n}-3$ fatty acids and atherosclerosis in Japanese, JapaneseAmerican, and white men: a cross-sectional study. J Am Coll Cardiol 2008, 52(6):417-424

11. Erkkila A, de Mello VD, Riserus U, Laaksonen DE: Dietary fatty acids and cardiovascular disease: an epidemiological approach. Prog Lipid Res 2008, 47(3):172-187.

12. Breslow JL: $\mathrm{n}-3$ fatty acids and cardiovascular disease. Am J Clin Nutr 2006, 83(6 Suppl):1477S-1482S

13. Ascherio A, Rimm EB, Stampfer MJ, Giovannucci EL, Willett WC: Dietary intake of marine $\mathrm{n}-3$ fatty acids, fish intake, and the risk of coronary disease among men. N Engl J Med 1995, 332(15):977-982.

14. Eckel RH, Grundy SM, Zimmet PZ: The metabolic syndrome. Lancet 2005, 365(9468):1415-1428

15. Balk E, Chung M, Lichtenstein A, Chew P, Kupelnick B, Lawrence A, DeVine D, Lau J: Effects of omega-3 fatty acids on cardiovascular risk factors and intermediate markers of cardiovascular disease. Evid Rep Technol Assess (Summ) 2004, 93: 1-6.

16. Wennberg $M$, Vessby B, Johansson I: Evaluation of relative intake of fatty acids according to the Northern Sweden FFQ with fatty acid levels in erythrocyte membranes as biomarkers. Public Health Nutr 2009, 12(9):1477-1484.

17. Stark KD: The percentage of $n-3$ highly unsaturated fatty acids in total HUFA as a biomarker for omega-3 fatty acid status in tissues. Lipids 2008, 43(1):45-53.

18. Chien $K L$, Lee BC, Hsu HC, Lin HJ, Chen MF, Lee YT: Prevalence, agreement and classification of various metabolic syndrome criteria among ethnic Chinese: A report on the hospital-based health diagnosis of the adult population. Atherosclerosis 2008, 196(2):764-771.

19. Chien KL, Hsu HC, Sung FC, Su TC, Chen MF, Lee YT: Metabolic syndrome as a risk factor for coronary heart disease and stroke: An 11-year prospective cohort in Taiwan community. Atherosclerosis 2007, 194(1):214-221.

20. Chien KL, Lin HJ, Lee BC, Hsu HC, Chen MF: Prediction model for high glycated hemoglobin concentration among ethnic Chinese in Taiwan. Cardiovascular Diabetology 2010, 9:59.

21. Chien KL, Hsu HC, Su TC, Lee YT: Consistency in genetic inheritance mode and heritability patterns of triglyceride vs. high density lipoprotein cholesterol ratio in two Taiwanese family samples. BMC Journal, Genetics 2003, 4:7-16

22. Grundy SM, Cleeman JI, Daniels SR, Donato KA, Eckel RH, Franklin BA, Gordon DJ, Krauss RM, Savage PJ, Smith SC Jr, et al: Diagnosis and management of the metabolic syndrome: an American Heart Association/National Heart, Lung, and Blood Institute Scientific Statement. Circulation 2005, 112(17):2735-2752.

23. Hosmer DW, Lemeshow S: The multiple logistic regression model. Applied logistic regression. 1 edition. New York: John Wiley \& Sons; 1989, 25-37.

24. Hanley JA, MCNeil BJ: A method of comparing the areas under receive operating characteristic curves derived from the same cases. Radiology 1983, 148:839-843

25. DeLong ER, DeLong DM, Clarke-Pearson DL: Comparing the areas under two or more correlated receiver operating characteristic curves: a nonparametric approach. Biometrics 1988, 44(3):837-845.

26. Pencina MJ, D' Agostino RBS, D' Agostino RBJ, Vasan RS: Evaluating the added predictive ability of a new marker: From area under the ROC curve to reclassification and beyond. Stat Med 2008, 27(2):157-172.

27. Cook NR: Use and misuse of the receiver operating characteristic curve in risk prediction. Circulation 2007, 115(7):928-935.

28. Ridker PM, Buring JE, Rifai N, Cook NR: Development and validation of improved algorithms for the assessment of global cardiovascular risk in women: the Reynolds Risk Score. Jama 2007, 297(6):611-619.

29. Yates J: External correspondence: decomposition of the mean probability score. Organizational Behavior and Human Performance 1982, 30:132-156. 
30. Schmid C, Griffith J: Multivariable classification rules: calibration and discrimination. In Encyclopedia of Biostatistics. Edited by: Armitage P, Colton T. Chichester, U.K.: Wiley; 1998.

doi:10.1186/1476-511X-10-33

Cite this article as: Chien et al:: Plasma fatty acids and the risk of metabolic syndrome in ethnic Chinese adults in Taiwan. Lipids in Health and Disease 2011 10:33.

Submit your next manuscript to BioMed Central and take full advantage of:

- Convenient online submission

- Thorough peer review

- No space constraints or color figure charges

- Immediate publication on acceptance

- Inclusion in PubMed, CAS, Scopus and Google Scholar

- Research which is freely available for redistribution 